\title{
Mechanisms of Redox Regulation of Chemoresistance in Tumor Cells by Phenolic Antioxidants
}

\author{
G. G. Martinovich ${ }^{a}$ * , I. V. Martinovich ${ }^{a}$, A. V. Vcherashniaya ${ }^{a}$, N. K. Zenkov ${ }^{b}$, \\ E. B. Menshchikova ${ }^{b, * *}$, N. V. Kandalintseva ${ }^{c, * * *}$, and S. N. Cherenkevich ${ }^{a}$ \\ ${ }^{a}$ Belarusian State University, Minsk, 220030 Belarus \\ ${ }^{b}$ Research Institute of Experimental and Clinical Medicine, Novosibirsk, 630117 Russia \\ ${ }^{c}$ Novosibirsk State Pedagogical University, Novosibirsk, 630126 Russia \\ *e-mail: martinovichgg@bsu.by \\ **e-mail:lemen@centercem.ru \\ ***e-mail: aquaphenol@mail.ru \\ Received June 7, 2017
}

\begin{abstract}
Effects of water-soluble sulfur-containing phenolic antioxidants sodium 3-(3'-tert-butyl-4'hydroxyphenyl)propyl thiosulfonate and potassium 3,5-dimethyl-4-hydroxybenzyl thioethanoate on chemoresistance in tumor cells have been studied. The studied phenolic antioxidants cause oppositely directed changes in the redox properties and chemoresistance in tumor cells. Potassium 3,5-dimethyl-4-hydroxybenzyl thioethanoate increases redox buffering capacity and doxorubicin resistance in tumor cells. Sodium 3-(3'tert-butyl-4'-hydroxyphenyl)propyl thiosulfonate reduces the redox buffering capacity, which leads to a decrease in the chemoresistance of tumor cells. These observations suggest that one of the key mechanisms responsible for the formation of tumor cell resistance to antitumor compounds is the attenuation of apoptosis through increase of redox buffering capacity. The dependence of protein sensor redox state on oxidant concentrations and on redox buffering capacity in cells has been determined based on the proposed biophysical model of redox-dependent mechanism of apoptosis activation.
\end{abstract}

Keywords: chemoresistance, sulfur-containing phenolic antioxidants, reactive oxygen species, tumor cells, redox state, redox signaling

DOI: $10.1134 / \mathrm{S} 000635091706015 \mathrm{X}$

The formation of resistance to chemotherapeutical drugs in tumor cells is a challenge of modern antitumor therapy. The investigation of mechanisms by which tumor cells respond to stress and the development of methods for diminishing their resistance to physical, chemical, and biological damaging factors is a topical task of medical biophysics.

Presently, it is recognized that the regulation of cell response to stress factors is mediated by the Keap1/Nrf2/ARE redox-dependent signaling system (Keap 1-Kelch-like ECH-associated protein 1, Nrf2-NF-E2-related factor 2, ARE-antioxidantresponse element) [1]. Transcription factor $\mathrm{Nrf} 2$ is the key element of the system. It governs the expression of

Abbreviations: Keap1, Kelch-like ECH-associated protein 1; Nrf2, NF-E2-related factor 2; ARE, antioxidant-response element; Klf9, Kruppel-like factor 9; ROS, reactive oxygen species; TS-13, sodium 3-(3'-tert-butyl-4'-hydroxyphenyl)propylthiosulfonate; BEP-11-K, potassium 3,5-dimethyl-4hydroxybenzyl thioethanoate; DCF, 2',7'-dichlorofluorescein; $\mathrm{H}_{2}$ DCF, 2',7'-dichlorodihydrofluorescein; NAC, N-acetyl-Lcysteine. genes whose promoter regions contain the ARE regulatory sequence [2]. At present, 500 such genes are known [3]. It has been found that the activation of the Keap1/Nrf2/ARE system via enhanced expression of ABCC1 (Mrp1), a key protein in ATP-dependent removal export of xenobiotics from cells, facilitates the development of multiple drug resistance in tumor cells $[4,5]$. The Keap1/Nrf2/ARE system also controls the key proteins of glutathione metabolism. Elevated glutathione concentrations help cells survive under stress conditions $[6,7]$.

When Nrf2 activation exceeds a certain threshold, genes whose products favor the development of oxidative stress are activated. At a high level of Nrf2 transcription activity, the level of Kruppel-like transcription factor 9 (Klf9) increases [8]. In turn, the interaction of Klf9 with DNA-binding sites modulates the expression of proteins involved in the regulation of the metabolism of reactive oxygen species (ROS), including NADPH oxidase [8]. Thus, Nrf2 is an important regulator of redox homeostasis in cells that is able to 


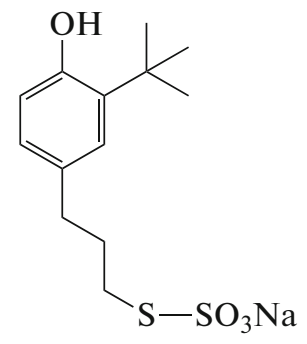

TS-13

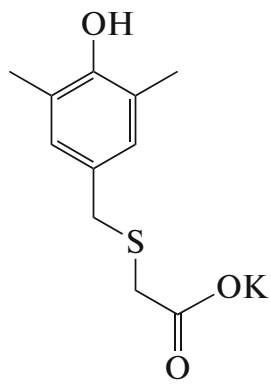

BEP-11-K<smiles>CC(=O)N[C@@H](CS)C(=O)O</smiles>

$\mathrm{N}$-acetyl-L-cysteine

Fig. 1. Structural formulas of antioxidants.

enhance both reducing and oxidative processes in cells.

The inactive Nrf2 transcription factor is noncovalently bound to the specific redox-dependent Keap1 protein. Modification of thiol groups of "key" cysteine residues in Keap1 by oxidation or electrophilic addition impairs Nrf2 ubiquitination and stability, transport to cell nucleus, and binding to ARE [2]. Nrf2 is activated by a variety of redox-active compounds, including natural and synthetic phenolic antioxidants. The ability of antioxidants to modulate Nrf2-driven transcription depends on their structure and function [9]. The indirect protective action of antioxidants in oxidative stress effected by Keap1/Nrf2/ARE is observed at concentrations of the components lower than those required for efficient scavenging of free radicals in a biologic medium [10].

It should be mentioned that only quinone forms of compounds that are formed by interaction of phenols with intracellular ROS activate ARE [11]. Therefore, intracellular ROS production may be considered as a key factor that determines the specific regulatory action of antioxidants. According to the modern concept of redox signaling, the effect of cell function regulation by ROS is determined not only by ROS type or concentrations but also by the type and intracellular concentrations of antioxidants and by the site of ROS formation [12]. Thus, the effect of redox-active compounds is determined by an entire set of components that form electron transport (redox) chains rather than by a single molecule; this depends on redox homeostasis parameters $[13,14]$.

Endogenous and exogenous antioxidants of similar structures may enter different electron transport chains and trigger different cell responses. We have shown that water-soluble sulfur-containing phenolic antioxidants 3-(3'-tert-butyl-4'-hydroxyphenyl)propyl thiosulfonate (TS-13) and potassium 3,5-dimethyl-4-hydroxybenzyl thioethanoate (BEP$11-\mathrm{K})$ induce oppositely directed responses in tumor cells $[14,15]$. The former induces tumor cell death via the mitochondrion-mediated pathway, whereas the latter stimulates proliferation of cultured tumor cells.
The ability of antioxidants to interact with Nrf2 suggests their involvement in the regulation of chemoresistance in tumor cells. However, the role of synthetic phenolic compounds in the formation of the drugs resistance in tumor cells has not been studied previously. Here, we investigate the action of water-soluble phenolics TS-13 and BEP-11-K on the chemoresistance of tumor cells and the role of the intracellular redox state in the mediation of action of these antioxidants and antitumor agents on tumor cells.

\section{MATERIALS AND METHODS}

The redox-active compounds examined in the study were water-soluble sulfur-containing phenolic antioxidants BEP-11-K and TS-13 synthesized at the Research Institute of Antioxidant Chemistry, Novosibirsk, Russia, and N-acetyl-L-cysteine (NAC), purchased from Sigma-Aldrich, United States. The structures of antioxidants are shown in Fig. 1.

Experiments were conducted with human larynx carcinoma HEp-2 cells. The cells were grown in DMEM medium (Sigma-Aldrich, United States) supplemented with $8-10 \%$ fetal bovine serum and $0.08 \mathrm{mg} / \mathrm{mL}$ gentamycin in an atmosphere with $5 \%$ $\mathrm{CO}_{2}$ at $37^{\circ} \mathrm{C}$. To determine the effects of the substances on the proliferation of tumor cells, a substance under study was applied to Petri dishes within $24 \mathrm{~h}$ after cell passage. Cells were counted after 3 days of growth. Proliferation activity was assessed from the proliferation index, which was taken as the ratio between the cell count after 4 days of growth and the number of inoculated cells. The sensitivity of modified cells to antitumor agents was assessed in their second passage without antioxidants. The percentage of surviving cells with reference to the control was determined. For preparation of the suspension, cells were flushed with trypsin-versene $1: 3(\mathrm{v} / \mathrm{v})$ after 6 days of growth.

Intracellular ROS production was assessed from the analysis of the oxidation rate of the fluorescent probe 2',7'-dichlorodihydrofluorescein $\left(\mathrm{H}_{2} \mathrm{DCF}\right)$ [16]. 
The quantitative characterization of changes in cell redox homeostasis was based on physicochemical parameters that we introduced: effective redox potential $\left(E^{\text {eff }}\right)$ and redox buffering capacity $(r)$. Their values were determined by redox titration based on the analysis of $\mathrm{H}_{2} \mathrm{DCF}$ oxidation with hydrogen peroxide added to the system $[17,18]$. The $E^{\text {eff }}$ parameter characterizes the overall ability of the multicomponent intracellular medium to donate or accept electrons. It is a quantitative measure of the work performed in transporting a unit charge in a multicomponent redox system [19]. The $r$ parameter quantitatively characterizes the ability of cells to resist the change of $E^{\text {eff }}$ caused by changes in the concentrations of oxidizing or reducing agents [18]. Taken together, these two parameters characterize the steady redox state in cells. The addition of an oxidant causes a transition from one state to another. The new steady state can be characterized with the equation $E^{\mathrm{eff}}=\Delta E_{0}^{\mathrm{eff}}+\Delta E^{\mathrm{eff}}$. The dependence of $\Delta E^{\mathrm{eff}}$ on the concentration of the added oxidant $c_{\mathrm{ox}}$ shown in double reciprocal coordinates is described by a linear equation with the slope $r=$ $k_{\text {cap }} / \Delta E_{\max }^{\text {eff }}$, which crosses the $Y$ axis at $1 / \Delta E_{\max }^{\text {eff }}$ and the $X$ axis at $-1 / k_{\text {cap }}$ :

$$
\frac{1}{\Delta E^{\mathrm{eff}}}=\frac{1}{\Delta E_{\max }^{\mathrm{eff}}}+\frac{k_{\mathrm{cap}}}{\Delta E_{\mathrm{max}}^{\mathrm{eff}}} \frac{1}{c_{\mathrm{ox}}},
$$

where $k_{\text {cap }}=\frac{\sum_{i=0}^{n} c_{i} z_{i}}{z_{\mathrm{ox}}}$.

Here $\Delta E_{\max }^{\mathrm{eff}}=E_{\mathrm{ox}}^{0^{\prime}}-\Delta E_{0}^{\mathrm{eff}}$ is the maximum change of the effective redox potential, which can happen at $c_{\mathrm{ox}} \gg c_{\text {red }} ; z_{\mathrm{ox}}$ is the number of electrons accepted by an oxidant molecule in transition to reduced state; $c_{i}$ is the concentration of the $i$ th redox-active molecule in biologic liquid; $z_{i}$ is the number of electrons accepted by the $i$ th oxidized molecule in transition to reduced form; $n$ is the number of various types of redox pairs involved in the formation of redox state; and $E_{\mathrm{ox}}^{0^{\prime}}$ is the redox potential of the oxidant at $\mathrm{pH}$ 7.0. The $k_{\text {cap }}$ constant has the dimensions of concentration and corresponds to the oxidant concentration at which the change of effective redox potential is half its maximum value.

This method is based on the experimentally and theoretically substantiated relationship between the $E^{\text {eff }}$ change induced by increased oxidant concentrations and the $\mathrm{H}_{2}$ DCF oxidation rate $\left(V_{\mathrm{f}}\right)$ [18]:

$$
V_{\mathrm{f}}=k_{0} \Delta E^{e \mathrm{ff}} .
$$

According to Eq. (2), the rate of 2', 7'-dichlorofluorescein (DCF) fluorescence intensity change is in direct proportion with the change of effective redox potential. Therefore, $r$ and $E^{\text {eff }}$ are determined from the analysis of the $V_{\mathrm{f}}$ vs. $c_{\text {ox }}$ plot obtained in experimental studies. It follows from Eqs. (1) and (2) that the maximum $V_{\mathrm{f}}^{\max }$ value decreases with an increase of the starting value of effective redox potential $E_{0}^{\text {eff }}$.

Measurements were carried out at $37^{\circ} \mathrm{C}$ in balanced salt buffer solution of the following composition: $131 \mathrm{mM} \mathrm{NaCl}, 5 \mathrm{mM} \mathrm{KCl}, 1.3 \mathrm{mM} \mathrm{CaCl}$, $1.3 \mathrm{mM} \mathrm{MgSO}_{4}, 0.4 \mathrm{mM} \mathrm{KH}{ }_{2} \mathrm{PO}_{4}, 20 \mathrm{mM}$ HEPES, and $6 \mathrm{mM}$ glucose, $\mathrm{pH}$ 7.4. The cell suspension was loaded with $\mathrm{H}_{2}$ DCF-DA (Sigma-Aldrich, United States) by incubating it with a $10 \mu \mathrm{M}$ probe in balanced buffered saline at $37^{\circ} \mathrm{C}$ for $45 \mathrm{~min}$. The fluorescence intensity of the DCF formed by $\mathrm{H}_{2}$ DCF oxidation was measured with an SM 2203 spectrofluorometer (SOLAR, Belarus) at the excitation wavelength $488 \mathrm{~nm}$ and emission wavelength $530 \mathrm{~nm}$.

The antitumor agents tested were doxorubicin (Belmedpreparaty, Belarus) and 2-isopropyl-5methyl-1,4-benzoquinone, also known as thymoquinone (Sigma-Aldrich, United States).

The kinetic plots presented in the paper are typical in a series of three to five independent experiments. The results are shown as the mean \pm the standard error of the mean for three to five replications. The confidence levels were determined by the Student's $t$-test and differences were considered significant at $p<0.05$.

\section{RESULTS AND DISCUSSION}

Proliferation of tumor cells under the action of antioxidants. To assess the influence of antioxidant on the proliferative activity of tumor cells, substances to be tested were applied at concentrations within 10$200 \mu \mathrm{M}$. This range corresponds to the physiological values of extracellular concentrations of endogenous low-molecular-weight water-soluble antioxidants. It was found that the magnitude and direction of the change of proliferative activity with the presence of antioxidants depended on antioxidant type (Fig. 2). The addition of $60 \mu \mathrm{M}$ BEP-11-K increased the proliferation index by $60 \pm 15 \%$. The same concentration of TS-13 reduced it by $50 \pm 12 \%$. Neither low $(60 \mu \mathrm{M})$ nor high $(1 \mathrm{mM})$ NAC concentrations altered the growth rate of cultured tumor cells.

The results indicate that only phenolic compounds affected tumor cell growth. Unlike NAC, TS-13 belongs to inducers of ARE-regulated genes; its action in tumor cells may be mediated by the Keap1/Nrf2/ARE system.

Changes of redox homeostasis in tumor cells exposed to antioxidants. The formation of the cell adaptive response involving transcription factor Nrf2 induces redox homeostasis changes. Therefore, at the next step of our study we quantitated redox homeostasis parameters in cells cultured in the presence of the antioxidants tested vs. control cells. 


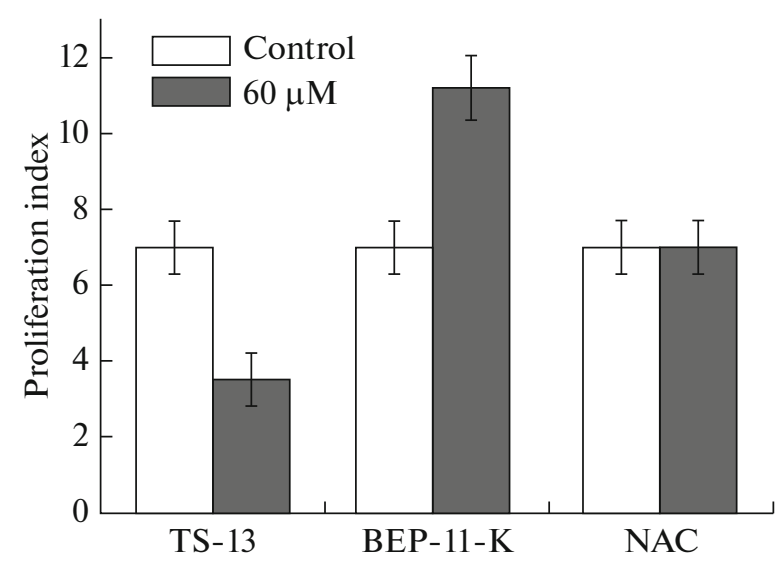

Fig. 2. Changes of the proliferative activity of HEp-2 cells with BEP-11-K, TS-13, and NAC. Antioxidant concentration-60 $\mu \mathrm{M}$.

We found that the amplitudes of changes of redox homeostasis parameters in cells cultured with different antioxidants differed significantly. Figure 3 a shows the variation of the fluorescence index $V_{\mathrm{f}}$ with the oxidant concentration in tumor cells cultured with antioxidants and control tumor cells in double reciprocal coordinates. Redox buffering capacitie and changes of the effective redox potential determined in experiments are shown in Figs. 3b and 3c.

Redox buffering capacity of cells grown with $60 \mu \mathrm{M}$ BEP-11-K increased by $50 \pm 10 \%$ and with $60 \mu \mathrm{M}$ TS-13, decreased by $50 \pm 10 \%$. The capacity of cells grown with $60 \mu \mathrm{M}$ NAC did not change. The effective redox potential of HEp-2 cells grown with BEP-11-K or NAC did not change, and the potential in the culture with TS-13 increased. Thus, changes of redox homeostasis indices in HEp-2 cultures are induced only with compounds that are presumed to be inducers of ARE-regulated genes; that is, BEP-11-K and TS-13. HEp-2 cells grown with TS-13 demonstrate more intense intracellular oxidation processes than control cells. This fact results in reduction of redox buffering capacity and elevation of effective redox potential. Culturing of cells with BEP-11-K elicits an adaptive response in cells, which brings about an increase in the content of intracellular reducing agents, which is apparent from the elevated redox buffering capacity. As the concentration of added antioxidants is considerably lower than that of endogenous antioxidants, the observed changes in redox state of the cells appear to be caused by activation of specific redox-signaling pathways.

In the context of the regulation mechanism that involves the Keap1/Nrf2/ARE signaling system, the opposite effects of BEP-11-K and TS-13 can be explained by their different abilities to regulate the action of the system. TS-13 is a potent Keap1/Nrf2/ARE inducer [20], whereas this function of BEP-11-K has not been studied. The decrease in
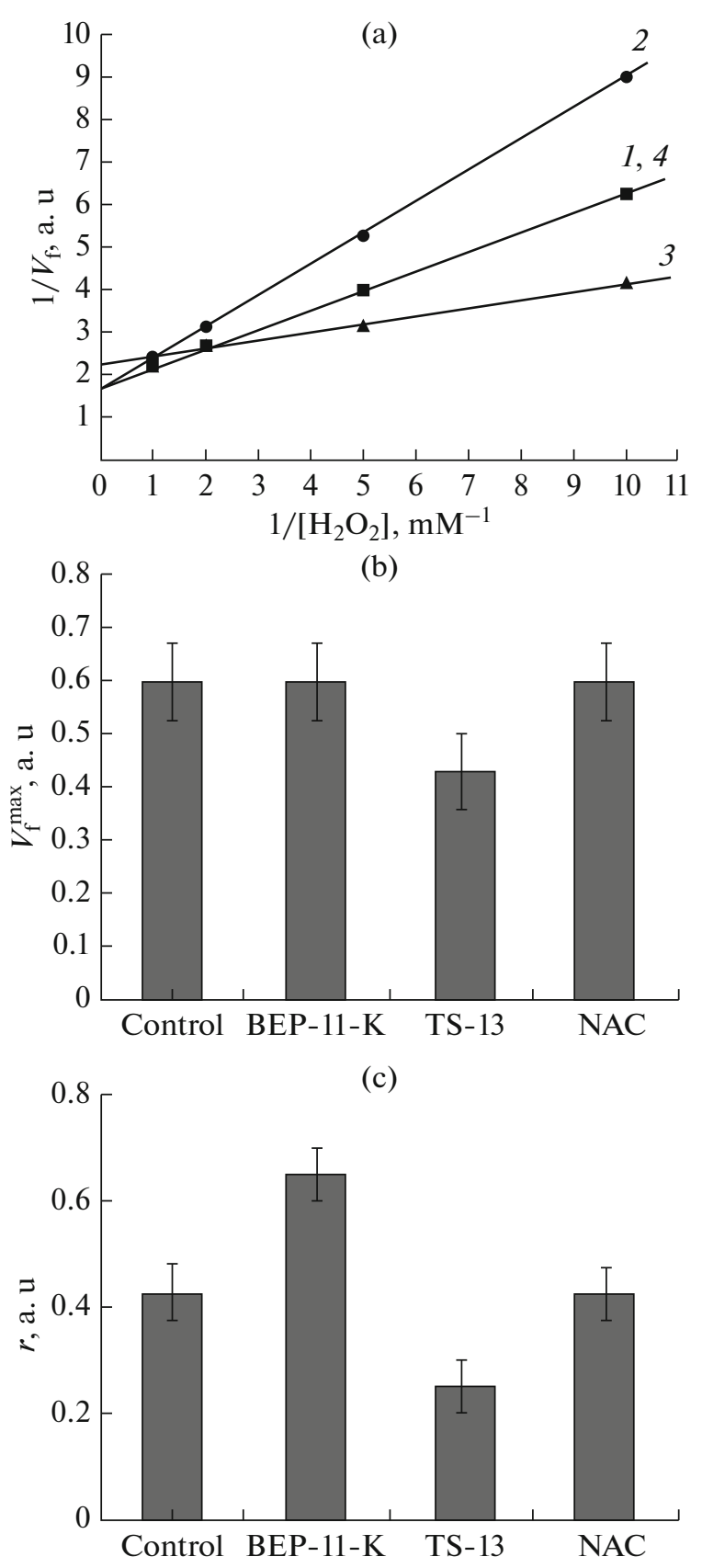

Fig. 3. Determination of redox homeostasis parameters in HEp- 2 cells. (a) The dependence of $V_{\mathrm{f}}$ on oxidant concentration in double reciprocal coordinates: (1) control; (2) cells cultured with $60 \mu \mathrm{M}$ BEP-11-K; (3) cells cultured with $60 \mu \mathrm{M}$ TS-13; (4) cells cultured with $60 \mu \mathrm{M}$ NAC. (b) The change of effective redox potential in control cells and in cells grown with antioxidants. (c) Redox buffering capacity in control cells and in cells grown with antioxidants.

redox buffering capacity and increase of the effective redox potential by TS-13 may stem from high ROS production in tumor cells as a result of the expression of transcription factor Klf9, induced by above-threshold Nrf2 activation and the expression of Klf9-regu- 
lated genes. Mutations that disrupt the function of Keap1 have been detected in carcinomas of various organs [21, 22]. As a result, constitutive Nrf2 activation is observed in many tumor tissues and cell lines [23]. It shares the bulk of the ambiguity of the action of exogenous Keap1/Nrf2/ARE regulators.

The action of TS-13, which is a Keap1/Nrf2/ARE inducer, results in crossing the Nrf2 activation threshold and upregulation of transcription factor Klf9 expression. In turn, the interaction of Klf9 with binding sites on DNA augments oxidative stress by modifying the expression of various proteins involved in the regulation of ROS metabolism [8]. With regard to the BEP-11-K structure, it is conceivable that the antioxidant induces low subthreshold Nrf2 activation, which elicits the adaptive response in cells, increasing their redox buffering capacity. However, we should keep in mind that the difference in the influence of the compounds on tumor cell proliferation recorded in our experiments may be explained by mediation of their effects by other redox-dependent signaling systems as well [24].

A biophysical model of the redox-dependent mechanism that induces apoptosis. Changes in redox parameters of cells affect redox signaling considerably. Thus they may modulate the functional properties and metabolism of cells. The concept of redox signaling combines intracellular processes of signal transduction, integrated through a series of electron-transport reactions that involve redox-active compounds, such as ROS and antioxidants [12, 25]. The molecular mechanism that regulates protein functions in redoxsignaling pathways is nonenzymatic oxidation of deprotonated thiol groups of cysteine residues in target proteins with modification of their catalytic or signaling functions. This mechanism is not confined to specific redox-active compounds. It can be triggered by both endogenous and exogenous electron acceptors. The intracellular location of systems producing ROS is an important factor determining their biologic action [26]. Previously, we showed that the ascorbate-dependent regulation of $\mathrm{Ca}^{2+}$ signaling included enhancement of local ROS production owing to the contribution of specific oxidoreductases: NADH:ubiquinone oxidoreductase (EC 1.6.5.3) and ubiquinol:cytochrome $c$ oxidoreductase (EC 1.10.2.2) [27]. Here, in addition to redox-active compounds and their targets, redox regulation involves oxidoreductase enzymes, whose location in the vicinity of target proteins determines the specific response of cells. In this model, the key item in the activation of specific redox-signaling mechanisms is the colocation of oxidoreductases involved in ROS production and ROS targets involved in the implementation of biologic response. We have shown that thymoquinone, which induces lower ROS production than 1,4-benzoquinone, is more toxic to tumor cells [28]. The higher thymoquinone toxicity is determined by the activation of redox-signaling mechanism triggering apoptosis, whose key element is the colocation of mitochondrial oxidoreductases involved in oxidant production and the corresponding redox sensor, as adenine nucleotide transporter. The functional state of the sensor is regulated by oxidation/reduction of thiol groups.

The primary free radical in mitochondria is superoxide anion radical $\left(\mathrm{O}_{2}^{--}\right)$. It is formed mainly in electron-transport chains by electron transfer from intermediate elements of the chain onto oxygen [29]. Dismutation of superoxide by $\mathrm{Mn}$-superoxide dismutase (EC 1.15.1.1) present in the mitochondrial matrix produces hydrogen peroxide. Presently, it is believed that hydrogen peroxide is the major ROS involved in regulatory processes [30]. The hydrogen peroxide formed in mitochondria causes oxidation of thiol groups in the adenine nucleotide transporter known as ADP/ATPtranslocase. Disulfide formation in this enzyme induces its binding to voltage-gated anion channel and cyclophilin D. The result is the formation of highly permeable pores and the launching of programmed cell death [31, 32].

In this model, protein thiols are regarded as nanoswitches in signal transduction according to the following reaction:

$$
\mathrm{H}_{2} \mathrm{O}_{2}+\mathrm{R}(\mathrm{SH})_{2} \rightarrow \mathrm{RSS}+2 \mathrm{H}_{2} \mathrm{O}
$$

where $\mathrm{R}(\mathrm{SH})_{2}$ is reduced form of protein thiols and RSS is a protein disulfide.

The start of apoptosis demands oxidation of a certain number of target thiol groups, which is the activation threshold for the process. As hydrogen peroxide also reacts with other reducing agents, whose concentrations and types determine the redox state of the intracellular medium, the concentration of oxidized SH groups in the sensor that form at the threshold inducer concentration depends on the intracellular concentration of reducing agents.

Hydrogen peroxide molecules can interact with $\mathrm{SH}$ groups of the sensor, giving rise to its active (oxidized) form, and with molecules of reducing agents, without altering the number of oxidized $\mathrm{SH}$ groups of the sensor. At higher reducing agent concentrations, the amount of oxidized SH groups of sensor would decrease; correspondingly, a decrease in the concentration of reducing agent would increase the number of sensor thiols oxidized by hydrogen peroxide. Thus, signal transduction mediated by hydrogen peroxide in the course of apoptosis induction would depend on redox state of intracellular environment of sensor.

As a first approximation, it may be assumed that the number of oxidized sensor thiols varies directly with the number of electrons $\left(Q_{\text {ox }}\right)$ transferred from $\mathrm{R}(\mathrm{SH})_{2}$ molecules to oxidant molecules. Again, the number of $\mathrm{R}(\mathrm{SH})_{2}$ molecules varies directly with the number of electrons $\left(Q_{\text {red }}\right)$ transferred from reducing molecules to oxidizing ones. This assumption can be expressed as follows: 


$$
\begin{gathered}
{[\mathrm{RSS}]=k Q_{\mathrm{ox}}=k z_{\mathrm{ox}} c_{\mathrm{ox}},} \\
{\left[\mathrm{R}(\mathrm{SH})_{2}\right]=k Q_{\mathrm{red}}=k \sum_{i=1}^{n} z_{i} c_{i},}
\end{gathered}
$$

where [RSS] is the concentration of oxidized $\mathrm{SH}$ groups of the sensor, $\left[\mathrm{R}(\mathrm{SH})_{2}\right]$ is the concentration of reduced $\mathrm{SH}$ groups of the sensor, $z_{\mathrm{ox}}$ is the number of electrons accepted by the oxidant molecule in conversion to the reduced form; $c_{\mathrm{ox}}$ is oxidant concentration; $z_{i}$ is the number of electrons transferred from the reducing molecule in oxidation; $c_{i}$ is the concentration of the $i$ th reducing agent; and $n$ is the number of various types of redox pairs contributing to the formation of redox state. Thus, redox state of the sensor can be determined as:

$$
\frac{[\mathrm{RSS}]}{\left[\mathrm{R}(\mathrm{SH})_{2}\right]}=\frac{z_{\mathrm{ox}} c_{\mathrm{ox}}}{\sum_{i=1}^{n} z_{i} c_{i}} .
$$

We assume that the overall sensor concentration $c_{0}$ does not change during the measurement, i.e.,

$$
[\mathrm{RSS}]+\left[\mathrm{R}(\mathrm{SH})_{2}\right]=c_{0}=\text { const. }
$$

Then, Eq. (5) can be rewritten as

$$
\frac{[\mathrm{RSS}]}{c_{0}-[\mathrm{RSS}]}=\frac{z_{\mathrm{ox}} c_{\mathrm{ox}}}{\sum_{i=1}^{n} z_{i} c_{i}}
$$

It follows from Eq. (7) that in the course of sensor oxidation the concentration of oxidized groups would change by

$$
[\mathrm{RSS}]=c_{0} \frac{z_{\mathrm{ox}} c_{\mathrm{ox}}}{z_{\mathrm{ox}} c_{\mathrm{ox}}+\sum_{i=1}^{n} z_{i} c_{i}},
$$

or

$$
[\mathrm{RSS}]=c_{0} \frac{1}{1+\frac{\sum_{i=1}^{n} z_{i} c_{i}}{z_{\mathrm{ox}} c_{\mathrm{ox}}}} .
$$
[13]:

Treating the process thermodynamically, we have

$$
\Delta E^{\mathrm{eff}}=c_{0} \frac{\Delta E_{\max }^{\mathrm{eff}}}{1+\frac{\sum_{i=1}^{n} z_{i} c_{i}}{z_{\mathrm{ox}} c_{\mathrm{ox}}}} .
$$

It follows from Eq. (9) with regard to Eq. (10) that redox sensors are sensors of changes in effective redox potential:

$$
[\mathrm{RSS}] \sim \Delta E^{\mathrm{eff}} .
$$

A change of hydrogen peroxide concentration causes a change of effective redox potential, which, in turn, changes redox potential of the sensor and triggers the cell response. In the action of an oxidant (hydrogen peroxide), the value of the induced change of effective redox potential depends on the redox buffering capacity.

The dependence of the redox buffering capacity on the concentration of redox-active compounds is as follows [17, 18]:

$$
r=\frac{\sum_{i=1}^{k} c_{i} z_{i}}{z_{\mathrm{ox}} \Delta E_{\mathrm{max}}^{\mathrm{eff}}} .
$$

It follows from Eqs. (10) and (14) that at low oxidant concentrations $c_{\mathrm{ox}}\left(c_{\mathrm{ox}} \ll \sum_{i=1}^{k} c_{i} z_{i}\right)$ the effective redox potential depends on oxidant concentration and redox buffering capacity as

$$
\Delta E^{\mathrm{eff}} \approx \frac{c_{\mathrm{ox}}}{r} .
$$

The activation of redox sensor and subsequent apoptosis induction occur when intracellular effective redox potential reaches a certain threshold. Consequently, they depend on redox buffering capacity. A decrease in redox buffering capacity lowers the threshold of apoptosis induction by endogenous and exogenous factors and an increase heightens it. Thus, a change in redox state alters the cell response.

In the experimental model, fluorescent probe $\mathrm{H}_{2}$ DCF also acts as a sensor of intracellular redox state. Kinetics of probe oxidation by hydrogen peroxide according to Eq. (15) should differ under different redox conditions. We demonstrated that kinetics of DCF fluorescence intensity formed after hydrogen peroxide addition varied significantly depending on whether the cells had been cultured with BEP-11-K, TS-13, or no antioxidant (Fig. 4). The decrease in redox buffering capacity and increase in effective redox potential seen in cells cultured with TS-13 accelerate $\mathrm{H}_{2} \mathrm{DCF}$ oxidation. Addition of BEP-11-K to tumor cell culture elicits a compensatory adapting response, which alters the intracellular concentration of redox-active compounds, primarily reducing agents. The increase in the concentration of reducing agents in HEp-2 cells cultured with BEP-11-K compared to unmodified cells decreases the amount of the probe oxidized by hydrogen peroxide, as apparent from the weakening of fluorescence intensity of DCF formed by $\mathrm{H}_{2} \mathrm{DCF}$ oxidation by hydrogen peroxide.

A similar effect is observed in ROS-mediated signal transduction. An increase in reducing agent content in cell decreases the level of ROS interacting with the target protein, thereby modifying cell response. According to Eqs. (11) and (15), with the action of substances inducing apoptosis according to the redoxdependent pathway, a $50 \%$ increase in cell redox buff- 


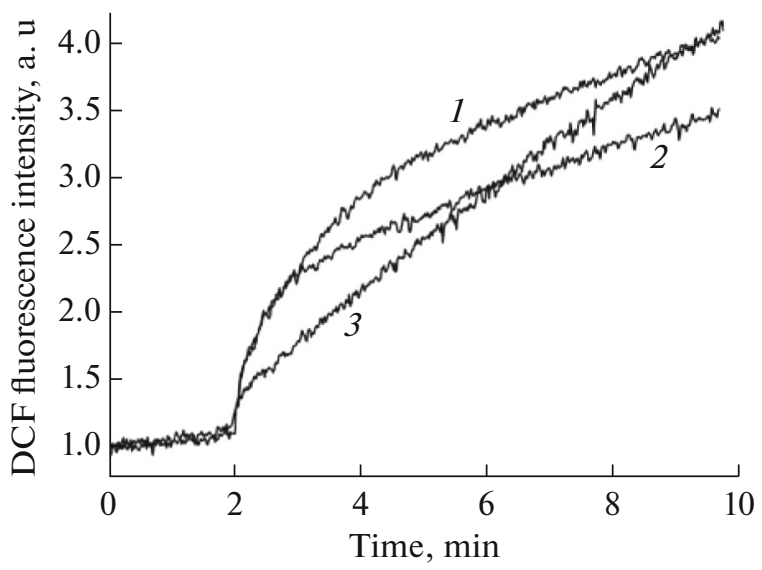

Fig. 4. The influence of $\mathrm{H}_{2} \mathrm{O}_{2}(100 \mu \mathrm{M})$ on 2', 7'-dichlorofluorescein fluorescence intensity in suspensions of HEp2 cells: (1) control; (2) cells cultured with $60 \mu \mathrm{M}$ BEP-11$\mathrm{K}$; (3) cells cultured with $60 \mu \mathrm{M}$ TS- 13 .

ering capacity would increase the number of surviving cells by about $50 \%$. Thus, according to our model, elevated redox buffering capacity of cells should attenuate apoptosis. Correspondingly, a reduction in redox buffering capacity should enhance apoptosis.

Apoptosis rescue is among the major cell mechanisms responsible for tumor cell resistance to treatment. Therefore, tumor cells that have been exposed to weaker Nrf2 inducers should show higher chemoresistance than control ones and, vice versa, cells treated with strong Nrf2 inducers should be less chemoresistant.

Chemoresistance of tumor cells exposed to antioxidants. We tested the chemoresistance of cells that were pretreated and not pretreated by antioxidants to the antitumor drug doxorubicin. Its concentration corresponding to $50 \%$ proliferation inhibition in the control cells (not treated with antioxidants) was $0.2 \mu \mathrm{M}$.

We showed that the change of the redox state of tumor cells increasing their redox buffering capacity was accompanied by greater cell resistance to antitumor agents. The number of cells that survived doxorubicin treatment in culture of cells treated with BEP$11-\mathrm{K}$ was $50 \%$ larger than in culture of cells not exposed to antioxidants (Fig. 5). Culturing of cells with NAC at low $(60 \mu \mathrm{M})$ or high $(1 \mathrm{mM})$ concentrations did not affect their doxorubicin resistance. It should be noted that cells cultured with BEP-11-K were resistant to other death-inducing agents as well. Previously, we showed that the action of thymoquinone, a biologically active compound of Nigella sativa, induced mitochondria-mediated apoptosis as a result of local increase of ROS production [28]. The toxic action of thymoquinone on cells cultured with $60 \mu \mathrm{M}$ $\mathrm{BEP}-11-\mathrm{K}$ prior to the passage was less pronounced than in control cells (Fig. 5).

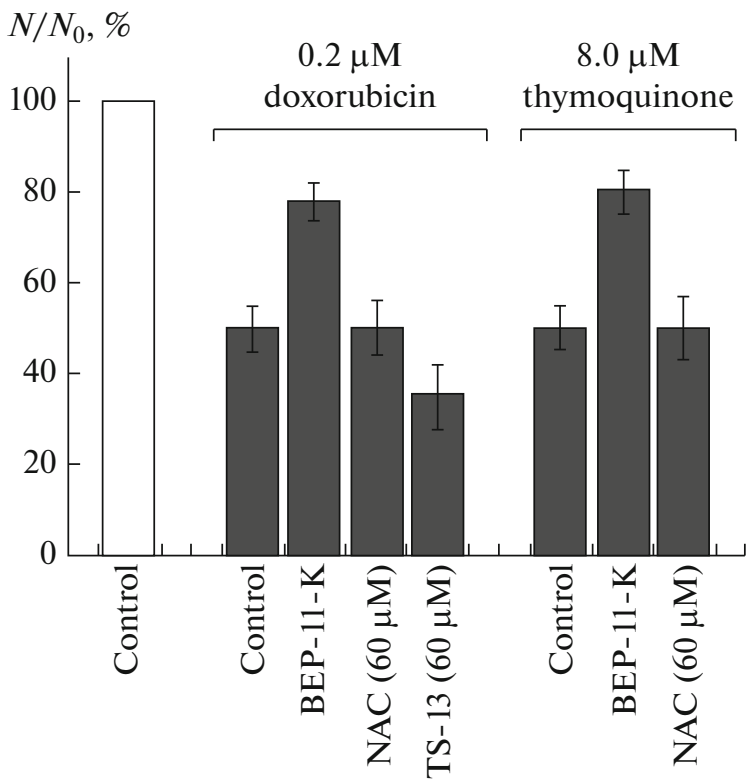

Fig. 5. The change in the number of HEp-2 cell cultured with doxorubicin and thymoquinone. BEP-11-K: cells were cultured with $60 \mu \mathrm{M}$ BEP-11-K prior to the passage; NAC $(60 \mu \mathrm{M})$ : cells were cultured with $60 \mu \mathrm{M}$ NAC prior to the passage; TS-13 $(60 \mu \mathrm{M})$ : cells were cultured with $60 \mu \mathrm{M}$ TS-13 prior to the passage.

It is known that treating a cell culture with chemotherapeutic agents at doses below the toxic threshold makes tumor cells more resistant to these agents. The inhibition of tumor cell growth by TS-13, as detected in our earlier study [15], is also indicative of its antitumor potential. Here, we showed that the TS-13induced decrease in redox buffering capacity decreases the resistance of tumor cells; this fact is an important advantage over conventional chemotherapeutic drugs. Figure 5 shows that the number of TS13-treated cells surviving doxorubicin treatment was less than in the culture of cells not exposed to antioxidants. Thus, our results indicate that BEP-11-K induces changes in redox homeostasis in tumor cells and these changes increase the resistance of tumor cells to antitumor agents. In contrast, redox homeostasis changes induced by TS-13 decrease this resistance.

The intrinsic and acquired resistance of tumor cells to cytostatic and cytotoxic drugs is the main cause of unsuccessful chemotherapy in many malignancies. Presently, attention is attracted to pharmacokinetic, metabolic, genetic, cellular, and other factors considered to be associated with tumor cell intrinsic resistance, fluctuating resistance, or lower sensitivity to cytostatic agents in refresher chemotherapy courses.

It is substantiated in our model that the change of redox status of cells by increasing the concentration of reducing agents weakens apoptosis induction and favors the development of drug resistance in tumor 
cells. We demonstrate that water-soluble sulfur-containing phenolic antioxidants are important regulators of the redox properties of tumor cells. The phenolic antioxidant BEP-11-K increases the redox buffering capacity and chemoresistance of tumor cells. Treating a culture with TS-13 causes a decrease in redox buffering capacity, which is accompanied by lower chemoresistance.

We note that the observed effects of antioxidants may be mediated by the specific redox-dependent Keap1/Nrf2/ARE signaling system. The cell-response type depends on the ability of antioxidants to regulate Nrf2 activity and on redox state of cell. The information on the involvement of Keap1/Nrf2/ARE in the induction and progress of neoplasms is controversial [33]. Based on the idea of autoprotective Nrf2 overexpression in tumor cells, it is conceivable that weak Nrf2 inducers elevate tumor cell resistance and cause an increase in proliferation rate, whereas strong inducers decrease the resistance and induce apoptosis.

The design of novel antioxidant drugs is an important field in modern pharmacology. However, the discovery of signaling pathways regulated by antioxidants demands that their mechanisms be investigated in more detail. Our studies revealed the ability of antioxidants to regulate the chemoresistance of tumor cells in a guided way; this opens new prospects in the development of antitumor technologies.

\section{ACKNOWLEDGMENTS}

This study was supported by the Belarusian Foundation for Basic Research, project M16P-022, and the Russian Foundation for Basic Research, project 1654-00050.

\section{REFERENCES}

1. T. W. Kensler, N. Wakabayashi and S. Biswal, Annu. Rev. Pharmacol. Toxicol. 47, 89 (2007).

2. N. K. Zenkov, E. B. Menshchikova, and V. O. Tkachev, Biochemistry (Moscow) 78 (1), 19 (2013).

3. N. K. Zenkov, A. V. Chechushkov, P. M. Kozhin, et al., Biochemistry (Moscow) 81 (4), 297 (2016).

4. C. Xu, C. Y. Li, and A. N. Kong, Arch. Pharm. Res. 28 (3), 249 (2005).

5. X. J. Wang, Z. Sun, N. F. Villeneuve, et al., Carcinogenesis 29, 1235 (2008).

6. X. Sun, H. Erb, and T. H. Murphy, Biochem. Biophys. Res. Commun. 326 (2), 371(2005).

7. M. Iida, S. Sunaga, N. Hirota, et al., J. Cancer Res. Clin. Oncol. 123, 619 (1997).
8. S. N. Zucker, E. E. Fink, A. Bagati, et al., Mol. Cell 53 (6), 916 (2014).

9. A. T. Dinkova-Kostova, J. W. Fahey, and P. Talalay, Meth. Enzymol. 382, 423 (2004).

10. L. Saso and O. Firuzi, Curr. Drug Targets 15 (13), 1177 (2014).

11. X. J. Wang, J. D. Hayes, L. G. Higgins, et al., Chem. Biol. 17 (1), 75 (2010).

12. S. N. Cherenkevich, G. G. Martinovich, I. V. Martinovich, et al., Proc. Nat. Acad. Science of Belarus, Ser. Biol. Sci. No. 1, 92 (2013).

13. G. G. Martinovich, I. V. Martinovich, and S. N. Cherenkevich, Biophysics (Moscow) 56 (3), 444 (2011).

14. G. G. Martinovich, I. V. Martinovich, E. B. Menshchikova, et al., Dokl. Nats. Akad. Nauk Belarusi 59 (3), 82 (2015).

15. G. G. Martinovich, I. V. Martinovich, N. K. Zenkov, et al., Biophysics (Moscow) 60 (1), 94 (2015).

16. G. G. Martinovich and S. N. Cherenkevich, Biomed. Khim. 51 (6), 626 (2005).

17. G. G. Martinovich, I. V. Martinovich, and S. N. Cherenkevich, Biophysics (Moscow) 53 (4), 618 (2008).

18. G. G. Martinovich, I. V. Martinovich, S. N. Cherenkevich, et al., Cell Biochem. Biophys. 58 (2), 75 (2010).

19. G. G. Martinovich, S. N. Cherenkevich, and H. Sauer, Eur. Biophys. J. 34 (7), 937 (2005).

20. E. Menshchikova, V. Tkachev, A. Lemza, et al., Inflammation Res. 63 (9), 729 (2014).

21. T. Ohta, K. Iijima, M. Miyamoto, et al., Cancer Res. 68, 1303 (2008).

22. L. M. Solis, C. Behrens, W. Dong, et al., Clin. Cancer Res. 16, 3743 (2010).

23. M. B. Sporn and K. T. Liby, Nat. Rev. Cancer 12, 564 (2012).

24. X. Gào and B. Schöttker, Oncotarget 8 (31), 51888 (2017). doi 10.18632/oncotarget.17128

25. D. P. Jones, J. Intern. Med. 268, 432 (2010).

26. L. S. Terada, J. Cell Biol. 174, 615 (2006).

27. G. G. Martinovich, E. N. Golubeva, I. V. Martinovich, et al., J. Biophys. 2012, 921653 (2012).

28. G. G. Martinovich, I. V. Martinovich, A. V. Vcherashniaya, et al., Biophysics (Moscow) 61 (6) 963 (2016).

29. S. Raha and B. H. Robinson, Trends Biochem. Sci. 25, 502 (2000).

30. H. Sies, Redox Biol. 11, 613 (2017).

31. A. P. Halestrap, K. Y. Woodfield, and C. P. Connern, J. Biol. Chem. 272, 3346 (1997).

32. G. Kroemer and J. C. Reed, Nat. Med. 6, 513 (2000).

33. L. Milkovic, N. Zarkovic, and L. Saso, Redox Biol. 12, 727 (2017).

Translated by Victor Gulevich

BIOPHYSICS Vol. 62 No. $6 \quad 2017$ 